Vol. 15, Núm. 4

Oct.-Dic. 2019. pp 222-230 doi: $10.35366 / 94048$

https://dx.doi.org/10.35366/94048

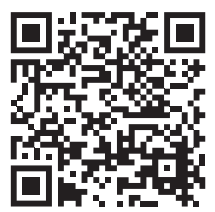

\title{
¿Qué hacer cuando la reversa falló? Protocolos de manejo. Revisión de alcance
}

\author{
What do you do when the reverse arthroplasty fails? Management protocols. Scope review
}

\author{
Roberto Enrique López Cervantes, * Josué Giovanni Escutia García, ${ }^{\ddagger}$ \\ José Máximo Gómez Acevedo§
}
* Cirujano Ortopedista y Traumatólogo, Cirujano Ortopedista en CFO (Clínica de fracturas por Osteoporosis), Sanatorio San Francisco de Asís Guadalajara, Jalisco, México. ORCID-id: 0000-0003-3808-1910
₹ Cirujano Ortopedista en Hospital Ángeles Morelia, Michoacán, México.
\& Cirujano Traumatólogo Ortopedista, Profesor de Ortopedia Universidad de Guadalajara, Cirujano Ortopedista en CFO (Clínica de fracturas por Osteoporosis), Sanatorio San Francisco de Asís Guadalajara, Jalisco, México.

Correspondencia: Dr. José Máximo Gómez Acevedo. CURAA (Centro de reconstrucción articular y artroscopia), Sanatorio San Francisco de Asís Guadalajara, Américas 1946 Col. Country Club Guadalajara, Jalisco, México.

Correo electrónico: maxgomeza@gmail.com

\section{RESUMEN}

Introducción: La artroplastia reversa es en la actualidad la artroplastia de hombro con más indicaciones y capacidad de solución de problemas. De todas las artroplastias reversas de hombro (ARH) más de $50 \%$ habrán fallado a los 10 años. El objetivo de esta revisión de alcance es identificar las principales problemáticas a las cuales nos enfrentamos al tener un paciente con una prótesis reversa fallida. Material y métodos: Se realizó una revisión de alcance del tema «fallo de prótesis reversa y revisión de prótesis reversa de hombro". Debido a la reciente aparición de este tipo de problemas y a su constante cambio en el manejo, se decidió realizarla de la literatura de los últimos cinco años (diciembre de 2014 a noviembre de 2019). Resultados: Las principales causas de revisión de la ARH son inestabilidad (32\%), infección (13.8\%), disociación del componente glenoideo (12.2\%), aflojamiento glenoideo (10.4\%), aflojamiento humeral $(5.5 \%)$, dificultad al poner la base glenoidea $(4.8 \%)$ y dificultad al colocar la glenoesfera (4.4\%). El abordaje diagnóstico

\section{ABSTRACT}

Introduction: Reverse arthroplasty is now the shoulder arthroplasty with the most indications and problem-solving capabilities. Of all RSAs (reverse shoulder arthroplasty), more than $50 \%$ will have failed within 10 years. The objective of this scope review is to identify the main problems we face when having a patient with a failed reverse prosthesis. Material and methods: A review of the scope of the issue of reverse prosthesis failure and review of reverse shoulder prosthesis was conducted. Due to the recent appearance of this type of problem and its constant change in management, it was decided to make it only from the literature of the last five years December 2014 to November 2019. Results: The main causes of RSA revision are instability (32\%), infection (13.8\%), dissociation of the glenoid component (12.2\%), glenoid loosening (10.4\%), humeral loosening (5.5\%), difficulty in placing the glenoid base (4.8\%), difficulty in placing the gleno-sphere (4.4\%). The complete diagnostic approach is vital in order to resolve 
completo es vital para poder resolver todas las complicaciones en una sola cirugía. En esta valoración se tendrán que tomar en cuenta cuatro factores principales: aflojamiento, pérdida ósea, acortamiento humeral y medialización glenoidea, y estado de los tejidos blandos. Se intentará colocar una $\mathrm{ARH}$ en la mayoría de los casos, lo cual es posible en $90 \%$ de las ocasiones, el resto tendrá que permanecer con algún espaciador o artroplastia de interposición. Conclusiones: La ARH fallida es una entidad que requiere de un cuidadoso abordaje diagnóstico y toma de decisiones individualizada. El tratamiento de todas las causas del fallo en una sola cirugía de revisión es vital para un resultado adecuado y para obtenerlo se intentará realizar la recolocación de una prótesis reversa en la mayoría de los casos.

Palabras clave: Artroplastia reversa, hombro, glenoides, prótesis de hombro, artroplastia de resección. all complications in a single surgery. Four main factors must be taken into account in this assessment: loosening, bone loss, humeral shortening, glenoid medialization and soft tissue condition. RSA will be attempted in most cases, which is possible $90 \%$ of the time, the rest will have to remain with some spacer or interposition arthroplasty. Conclusions: Failed RSA is an entity which requires a careful diagnostic approach and individualized decision making. Treatment of all causes of failure in a single revision surgery is vital for an adequate outcome. To obtain an adequate result, a reverse prosthesis will be attempted in most cases.

Keywords: Reverse arthroplasty, shoulder, glenoid, shoulder prosthesis, resection arthroplasty.

\section{INTRODUCCIÓN}

La artroplastia reversa es en la actualidad la artroplastia de hombro con más indicaciones y capacidad de solución de problemas, desde su indicación inicial que fue la artropatía por degeneración del mango rotador hasta en la actualidad el tratamiento de las fracturas del húmero proximal, en el cual su uso ha aumentado en $12-18 \%$ de 2007 a 2016 en Alemania. ${ }^{1}$

Así como su uso incrementa, la cantidad de complicaciones y revisiones también. La artroplastia reversa de hombro $(\mathrm{ARH})$ tiene una supervivencia promedio a 10 años de $58-76 \%,{ }^{2}$ por ende, la tasa de revisiones tenderá a subir en los próximos años. Es por ello que este aumento nos obliga a entender más sus causas de fallo y opciones de manejo.

En el caso de presentar una o varias de estas complicaciones en la artroplastia reversa, lo primero será identificar todas las complicaciones que el caso pueda presentar para solamente realizar una cirugía de revisión.

El objetivo de este trabajo es identificar las principales problemáticas a las cuales nos enfrentamos al tener un paciente con una prótesis reversa fallida, conocer las complicaciones que podemos tener y esclarecer las principales características a tomar en cuenta para poder realizar una estrategia de tratamiento adecuada y llegar a una resolución exitosa en la mayoría de los casos.

\section{MATERIAL Y MÉTODOS}

Se realizó una revisión de alcance del tema «fallo de prótesis reversa y revisión de prótesis reversa de hombro». Debido a la reciente aparición de este tipo de 
problemas y a su constante cambio en el manejo, se decidió realizarla de la literatura de los últimos cinco años (diciembre de 2014 a noviembre de 2019).

La revisión de alcance de la literatura se basó en la metodología establecida por Colquhoun. ${ }^{3}$ Se realizó la búsqueda en el idioma inglés de los términos «revisión de reversa», "artroplastia de resección» y «fallo de prótesis reversa» en las bases de datos PubMed, Ovid, ScienceDirect y Cochrane.

Finalmente, se realizó una descripción del tratamiento basado en la literatura revisada, plasmado de manera narrativa para un adecuado entendimiento del mismo, dividido en subtemas para abordar de una manera sistemática cada uno de los problemas a los que nos enfrentamos.

\section{RESULTADOS}

Se obtuvieron 4,631 publicaciones indexadas. Después de excluir las publicaciones no referentes al tema, duplicadas, con antigüedad mayor a cinco años, escritas en idiomas distintos a inglés y español y cartas al editor, se incluyeron 26 publicaciones y tres referencias cruzadas, de las cuales fueron: dos metaanálisis, cuatro revisiones sistemáticas, 10 artículos de revisión, siete cohortes prospectivas y seis series de casos.

En un estudio de 2,390 artroplastias reversas se observó que las principales causas de revisión son: inestabilidad (32\%), infección (13.8\%), disociación del componente glenoideo (12.2\%), aflojamiento glenoideo (10.4\%), aflojamiento humeral (5.5\%), dificultad al poner la base glenoidea (4.8\%) y dificultad al colocar la glenoesfera (4.4\%). 4,5

En todos los casos en los cuales se sospeche de fallo en la artroplastia reversa, ya sea por dolor, pérdida de la función, luxación, rigidez o presencia de fístula, se deberá realizar un abordaje diagnóstico extenso.

Durante nuestro abordaje diagnóstico tendremos que buscar intencionadamente las principales causas de revisión. Se describe que el abordaje diagnóstico debe ser protocolizado y completo en todos los casos. En esta valoración se tendrán que tomar en cuenta cuatro factores principales:

- Aflojamiento e infección.

- Pérdida ósea humeral/glenoidea.

- Acortamiento humeral/medialización glenoidea.

- Estado de los tejidos blandos.

Con el objetivo de poder estudiar de manera adecuada al paciente se necesitará una exploración física completa, validando las características del deltoides, la presencia de restricciones fijas de la movilidad, características de los rotadores del hombro (subescapular, pectoral mayor, infraespinoso y redondo menor). Además, la valoración de la integridad y fuerza de los rotadores internos y externos es necesaria para valorar la necesidad de transferencias musculares. ${ }^{2}$

Nuestra valoración clínica deberá estar acompañada de algunos exámenes paraclínicos mínimos como:2,6 
- Radiografía AP verdadera en rotación neutra de ambos hombros.

- Escanometría con cuadrícula humeral bilateral.

- Tomografía computarizada con supresión metálica del hombro afectado.

- Exámenes de sangre como la biometría hemática, velocidad de sedimentación globular, proteína $\mathrm{C}$ reactiva, dímero $\mathrm{D}$. $^{7}$

Hay que tomar en cuenta que en lo común un solo caso de cirugía fallida vendrá acompañado de varios problemas juntos, requiriendo la solución de todos ellos en una sola cirugía.

\section{Inestabilidad protésica}

Es la primera causa de fallo, distinguimos la inestabilidad por la presencia de una luxación protésica. Ésta a su vez la podemos dividir para su tratamiento en dos tipos de acuerdo con Bouleau: temprana (primeros tres meses) y tardía (más de tres meses). ${ }^{6}$

En la inestabilidad temprana aún no se tiene pérdida ósea. Una vez descartado un aflojamiento por infección, se podrá realizar una reducción cerrada e inmovilización con un cabestrillo en abducción por 4-6 semanas. Esto con una efectividad de 30 a $50 \% .^{6}$

En caso de que esto falle en el tratamiento conservador o en todos los casos de luxaciones tardías, habrá que verificar nuevamente nuestro abordaje diagnóstico poniendo especial atención en la altura humeral, la medialización glenoidea, la rotación humeral, el offset humeral y el estado de los tejidos blandos.

En la malrotación del componente humeral es indispensable la remoción del implante y su recolocación. En la mayoría de los casos el problema está relacionado con acortamiento humeral o medialización glenoidea, como menciona $\mathrm{P}$ Boileau; 6 en caso de detectar un acortamiento habrá que medirlo con base en el hombro contralateral.

En acortamientos menores de $15 \mathrm{~mm}$ el manejo, una vez que se asegura una adecuada rotación humeral, podrá hacerse con alzas metálicas, aumento del tamaño del polietileno o colocar una glenoides más grande. Mientras que en acortamientos mayores de $15 \mathrm{~mm}$ el manejo debe ser recambiando el componente humeral. ${ }^{8}$

\section{Infecciones}

En el registro danés de artroplastia reversa de hombro, las infecciones fueron la primera causa de revisión (44\%). Cincuenta y uno por ciento de las revisiones se realizaron en los primeros seis meses y un factor de riesgo para infección fue el ser paciente masculino..$^{9,10}$

En hombro los principales agentes son el Propionibacterium acnes, Staphylococcus epidermidis y Staphylococcus aureus. Algunos de ellos causan una infección de bajo grado, silente y crónica, por lo cual al obtener un cultivo por aspiración debemos esperar hasta 21 días para descartar la formación de Propionibacterium acnes. ${ }^{11}$ 
En las infecciones agudas (menores a tres meses) es efectivo el tratamiento con desbridamiento quirúrgico, recambio de glenoesfera e inserto humeral, y antibioticoterapia por periodos prolongados de hasta dos años. ${ }^{6,12}$

En las infecciones crónicas (mayores a tres meses) existen dos opciones:

- Artroplastia de resección o colocación de espaciadores: recomendado en pacientes frágiles o en sujetos con intentos previos de revisión. Se advierte un resultado funcional pobre.

- Revisión de artroplastia en uno o dos tiempos: en el caso de ya conocer el germen y haber instaurado antibioticoterapia específica se podrá realizar en un tiempo. ${ }^{13}$ En caso contrario, se dejará un espaciador en un periodo de tres hasta siete meses, así como antibioticoterapia específica hasta obtener marcadores de infección negativos, para posteriormente realizar una nueva planificación preoperatoria y valorar la posibilidad de una artroplastia reversa de revisión. ${ }^{14}$

Los resultados funcionales en las cirugías de revisión por infección nunca son tan halagadores. Hasta $38 \%$ de los pacientes tienen alguna complicación posterior. Los resultados funcionales y de efectividad de erradicación de la infección se muestra en la Tabla 1. ${ }^{13,15}$

\section{Pérdida ósea glenoidea y aflojamiento aséptico}

El aflojamiento óseo glenoideo puede darse por tres principales causas que hay que identificar: la presencia de infección, una mala colocación inicial y por resorción del injerto. ${ }^{6}$

Es más frecuente el aflojamiento glenoideo en los casos de cirugía de revisión de reversa y cirugía de osteoartritis primaria con pérdida ósea. ${ }^{16}$

Cuando tenemos una resorción ósea aséptica o del injerto hay que recordar que la posición ideal de la glenoesfera debe ser lo más inferior posible y con una inclinación inferior de $10^{\circ} .{ }^{17}$ Una posición muy arriba de la glenoesfera o con inclinación superior a los 10 grados que nos puede llevar al notching. ${ }^{18}$

Tabla 1: Efectividad de tratamientos en cirugía por infección periprotésica.

\begin{tabular}{lcc} 
Procedimiento realizado & Funcionalidad CMS & Erradicación de infeccio \\
\hline Artroplastia de revisión en 1 tiempo & $49.7-51$ & $94.7-96$ \\
Artroplastia de revisión en 2 tiempos & $44-45.3$ & $86-90.8$ \\
Antibioticoterapia y desbridamiento & 42.8 & 65 \\
Colocación de espaciador & $31-33.8$ & $93-95.6$ \\
Artroplastia de resección & $32-28.8$ & $85-86.7$
\end{tabular}

CMS $=$ Constant Murley Score 
Cuando tenemos un defecto glenoideo lo podemos dividir en tres grupos según $\mathrm{P}$ Boileau:

- Cavitario: cuando tenemos integridad de las paredes anterior y posterior.

- No contenible con defecto de una pared: por lo común la pared posterior es la más afectada.

- Defecto completo (afectación de las dos paredes): en estos casos se recomienda una planeación cuidadosa de los defectos mediante tomografía, posterior a haber retirado el implante previo.

En los casos en lo que se observa aflojamiento glenoideo con o sin migración, con ausencia de dolor o con alteración funcional mínima, se ha visto que el tratamiento conservador ofrece similar resultado a la revisión de glenoesfera temprana. ${ }^{19}$

- Los defectos cavitarios se pueden realizar con impactación de hueso esponjoso o fragmento de hueso esponjoso. En los no contenibles con defecto de una pared se utiliza autoinjerto tricortical de moldeado a medida, teniendo como opción la reconstrucción con alzas metálicas. ${ }^{2}$ En los defectos completos (afectación de las dos paredes): en estos casos se recomienda una planeación cuidadosa de los defectos mediante tomografía. En la actualidad existen programas, los cuales nos permiten realizar una reconstrucción exacta del defecto y un implante personalizado para cada caso, como lo es el sistema Glenius..$^{20}$ Se pueden resolver al colocar aloinjerto o autoinjerto, se da la inclinación inferior deseada y se quita la retroversión excesiva encontrada en algunos casos, con un índice de resorción del injerto de hasta $25 \%{ }^{17,21,22}$ Debemos tomar en cuenta que la fijación de la glenoesfera se tiene que hacer con un Peg central largo que se fije al menos $1 \mathrm{~cm}$ dentro de la glena nativa.

\section{Aflojamiento humeral}

El aflojamiento humeral es una complicación que se observa con relativa frecuencia en la $\mathrm{ARH}$, hay algunos factores que nos predisponen a la misma: ${ }^{6}$

- Pérdida ósea humeral

- Ausencia de tuberosidad mayor

- Osteoporosis

- Paciente joven y activo

- En las ARH por fractura

- Notching escapular

- Infección

En todo aflojamiento humeral la remoción del implante no será difícil, lo que se debe planificar a detalle es la reconstrucción, para darle un volumen y longitud adecuada. 
La altura de la prótesis debe estar dada por la tensión de los tejidos blandos y la estabilidad de la prótesis, así como la medición del húmero contralateral. ${ }^{23}$

Como señaló P Boileau, ${ }^{6}$ podemos dividir los defectos en dos tipos:

- Menores a $5 \mathrm{~cm}$ y más de $5 \mathrm{~cm}$.

El tratamiento de los defectos humerales podrá realizarse con recambio a un componente de mayor tamaño y/o bloqueado, describiéndose también el uso suplementario de collares de cemento o aloinjerto humeral. Este tipo de reconstrucción tiene una tasa de complicaciones de $36 \%$ a cinco años. ${ }^{24} \mathrm{La}$ reconstrucción tendrá que evitar el exceso de fuerzas rotacionales en valgo y mejorar la fuerza de coaptación del deltoides «wrapping angle», lo cual mejora la estabilidad. ${ }^{6}$

En los defectos humerales masivos se describe la reconstrucción con aloinjerto y fijación del aloinjerto mediante una placa bloqueada con técnica de compresión interfragmentaria y posteriormente se realiza la cementación del componente al húmero y aloinjerto. ${ }^{25}$

Durante la realización de estas técnicas la incidencia aproximada de fracturas humerales es de $16 \%$, por lo que debemos estar preparados para su manejo. ${ }^{11}$

En los casos en que el manto previo de cemento esté bien fijo al húmero, se puede utilizar la técnica "cement within cement», ${ }^{26}$ técnica descrita por Wagner, Sperling y Sánchez-Sotelo. En esta técnica se utiliza el manto de cemento del vástago previo, solamente preparándolo con un legrado del mismo, para después poder colocar un vástago corto cementado dentro del mismo.

En todos los casos se intentará su revisión con otra prótesis reversa, siendo ésta posible hasta en $90 \%$ de los casos, en el otro $10 \%$ se tendrá que realizar una artroplastia de resección o la colocación de un espaciador. ${ }^{27}$ Hasta $59 \%$ de los pacientes a quien se les realice un recambio de prótesis reversa podrán ser libres de alguna complicación, ${ }^{28} 22-33 \%$ requerirán alguna otra cirugía y $19 \%$ tendrá algún otro tipo de complicaciones, las cuales no requerirán de otra cirugía. ${ }^{2}$

\section{Artroplastia de resección o espaciador}

El uso de la artroplastia de resección, uso de espaciador o conversión a hemiprótesis sólo se recomienda en los casos en los que no es posible la reconstrucción, el paciente tiene una infección multirresistente o es un paciente muy frágil. 6

La artroplastia de resección ha demostrado disminuir el dolor de seis a tres en una escala visual análoga (EVA). Sin embargo, también disminuye la capacidad funcional. ${ }^{29}$

En la artroplastia de resección se tendrá que retirar el total de material protésico, así como el cemento humeral y glenoideo. ${ }^{29}$ 


\section{CONCLUSIONES}

La ARH fallida es una patología cada vez más frecuente; debido a la cantidad de indicaciones de este tipo de artroplastia el análisis de la misma requiere un cuidadoso abordaje diagnóstico y la utilización de varios estudios paraclínicos, con el objetivo de determinar todas las causas del fallo, ya que el denominador común es encontrar varios problemas en un solo caso.

En el caso de las infecciones debemos poner especial atención en aquéllas de bajo grado, debido a su elevada frecuencia en este tipo de cirugías.

En el tratamiento de una prótesis reversa fallida se intentará realizar una artroplastia reversa de revisión, se debe tomar en cuenta que la solución de todas las causas del fallo en una sola cirugía de revisión es vital para un resultado adecuado.

En caso de que la cirugía de revisión no sea posible, se realizará la artroplastia de resección.

Conflicto de intereses: Estos autores declaran que no tienen conflicto de intereses.

\section{BIBLIOGRAFÍA}

1. Klug A, Gramlich Y, Wincheringer D, Schmidt-Horlohé K, Hoffmann R. Trends in surgical management of proximal humeral fractures in adults: a nationwide study of records in Germany from 2007 to 2016. Arch Orthop Trauma Surg. 2019; 139 (12): 1713-1721. doi: 10.1007/s00402019-03252-1.

2. Chalmers PN, Boileau P, Romeo AA, Tashjian RZ. Revision reverse shoulder arthroplasty. J Am Acad Orthop Surg. 2019; 27 (12): 426-436. doi: 10.5435/JAAOS-D-17-00535.

3. Colquhoun HL, Levac D, Brien KKO, et al. Scoping reviews: time for clarity in definition, methods, and reporting. J Clin Epidemiol. 2014; 67 (12): 1291-1294. doi: 10.1016/j.jclinepi.2014.03.013.

4. Somerson JS, Hsu JE, Neradilek MB, Matsen FA. Analysis of 4063 complications of shoulder arthroplasty reported to the US Food and Drug Administration from 2012 to 2016. J Shoulder Elbow Surg. 2018; 27 (11): 1978-1986. doi: 10.1016/j.jse.2018.03.025.

5. Stephens BC, Simon P, Clark RE, et al. Revision for a failed reverse: A 12-year review of a lateralized implant. J Shoulder Elbow Surg. 2016; 25 (5): e115-e124. doi: 10.1016/j.jse.2015.09.027.

6. Boileau P. Complications and revision of reverse total shoulder arthroplasty. Orthop Traumatol Surg Res. 2016; 102 (1): S33-S43. doi: 10.1016/j.otsr.2015.06.031.

7. Shohat N, Tan TL, Della Valle CJ, et al. Development and validation of an evidence-based algorithm for diagnosing periprosthetic joint infection. J Arthroplasty. 2019; 34 (11): 2730-2736.e1. doi: 10.1016/j. arth.2019.06.016.

8. Chae J, Siljander M, Michael Wiater J. Instability in reverse total shoulder arthroplasty. J Am Acad Orthop Surg. 2018; 26 (17): 587-596. doi: 10.5435/JAAOS-D-16-00408.

9. Lehtimäki K, Rasmussen JV, Mokka J, et al. Risk and risk factors for revision after primary reverse shoulder arthroplasty for cuff tear arthropathy and osteoarthritis: a Nordic Arthroplasty Register Association study. J Shoulder Elbow Surg. 2018; 27 (9): 1596-1601. doi: 10.1016/j.jse.2018.02.060.

10. Moeini S, Rasmussen JV, Salomonsson B, et al. Reverse shoulder arthroplasty has a higher risk of revision due to infection than anatomical shoulder arthroplasty. Bone Jt J. 2019; 101 B (6): 702-707. doi: 10.1302/0301-620X.101B6.BJJ-2018-1348.R1.

11. Petkovic $D$, Kovacevic $D$, Levine WN, Jobin $C M$. Management of the failed arthroplasty for proximal humerus fracture. J Am Acad Orthop Surg. 2019; 27 (2): 39-49. doi: 10.5435/JAAOS-D-17-00051.

12. Kunutsor SK, Beswick AD, Whitehouse MR, Wylde V, Blom AW. Debridement, antibiotics and implant retention for periprosthetic joint infections: A systematic review and meta-analysis of treatment outcomes. J Infect. 2018;77(6):479-488. doi:10.1016/j.jinf.2018.08.017.

13. Nelson GN, Davis DE, Namdari S. Outcomes in the treatment of periprosthetic joint infection after shoulder arthroplasty: a systematic review. J Shoulder Elbow Surg. 2016; 25 (8): 1337-1345. doi: 10.1016/j.jse.2015.11.064. 
14. Grubhofer F, Imam MA, Wieser K, Achermann Y, Meyer DC, Gerber C. Staged revision with antibiotic spacers for shoulder prosthetic joint infections yields high infection control. Clin Orthop Relat Res. 2018; 476 (1): 146-152. doi: 10.1007/s11999.0000000000000049.

15. George DA, Volpin A, Scarponi S, Haddad FS, Romanò CL. Does exchange arthroplasty of an infected shoulder prosthesis provide better eradication rate and better functional outcome, compared to a permanent spacer or resection arthroplasty? a systematic review Orthopedics and biomechanics. BMC Musculoskelet Disord. 2016; 17 (1): 1-9. doi: 10.1186/s12891-016-0901-6.

16. Rojas J, Choi K, Joseph J, Srikumaran U, McFarland EG. Aseptic Glenoid Baseplate Loosening After Reverse Total Shoulder Arthroplasty: A Systematic Review and Meta-Analysis. JBJS Rev. $2019 ; 7$ (5): e7. doi: 10.2106/JBJS.RVW.18.00132.

17. Boileau P, Morin-Salvo N, Gauci MO, et al. Angled BIO-RSA (bony-increased offset-reverse shoulder arthroplasty): a solution for the management of glenoid bone loss and erosion. J Shoulder Elbow Surg. 2017; 26 (12): 2133-2142. doi: 10.1016/j.jse.2017.05.024.

18. Friedman RJ, Barcel DA, Eichinger JK. Scapular notching in reverse total shoulder arthroplasty. J Am Acad Orthop Surg. 2019; 27 (6): 200-209. doi: 10.5435/JAAOS-D-17-00026.

19. Lädermann A, Schwitzguebel AJ, Edwards TB, et al. Glenoid loosening and migration in reverse shoulder arthroplasty. Bone Joint J. 2019; 101-B (4): 461-469. doi: 10.1302/0301-620x.101b4.bjj-2018-1275.r1.

20. Debeer P, Berghs B, Pouliart N, Van den Bogaert G, Verhaegen F, Nijs S. Treatment of severe glenoid deficiencies in reverse shoulder arthroplasty: the Glenius Glenoid Reconstruction System experience. J Shoulder Elbow Surg. 2019; 28 (8): 1601-1608. doi: 10.1016/j.jse.2018.11.061.

21. Ho JC, Thakar O, Chan WW, Nicholson T, Williams GR, Namdari S. Early radiographic failure of reverse total shoulder arthroplasty with structural bone graft for glenoid bone loss. J Shoulder Elbow Surg. 2020; 29 (3): 550-560. doi: 10.1016/j.jse.2019.07.035.

22. Tashjian RZ, Broschinsky K, Stertz I, Chalmers PN. Structural glenoid allograft reconstruction during reverse total shoulder arthroplasty. J Shoulder Elbow Surg. 2020; 29 (3): 534-540. doi: 10.1016/j. jse.2019.07.011.

23. Stephens SP, Paisley KC, Giveans MR, Wirth MA. The effect of proximal humeral bone loss on revision reverse total shoulder arthroplasty. J Shoulder Elbow Surg. 2015; 24 (10): 1519-1526. doi: 10.1016/j. jse.2015.02.020.

24. Werner BS, Abdelkawi AF, Boehm D, et al. Long-term analysis of revision reverse shoulder arthroplasty using cemented long stems. J Shoulder Elbow Surg. 2017; 26 (2): 273-278. doi: 10.1016/j. jse.2016.05.015.

25. Sanchez-Sotelo J, Wagner ER, Houdek MT. Subspecialty procedures: Allograft-prosthetic composite reconstruction for massive proximal humeral bone loss in reverse shoulder arthroplasty. JBJS Essent Surg Tech. 2018; 8 (1): 2069-2076. doi: 10.2106/JBJS.ST.17.00051.

26. Wagner ER, Houdek MT, Hernandez NM, Cofield RH, Sánchez-Sotelo J, Sperling JW. Cement-withincement technique in revision reverse shoulder arthroplasty. J Shoulder Elbow Surg. 2017; 26 (8): 1448-1453. doi: 10.1016/j.jse.2017.01.013.

27. Gauci MO, Cavalier M, Gonzalez JF, et al. Revision of failed shoulder arthroplasty: epidemiology, etiology, and surgical options. J Shoulder Elbow Surg. 2020; 29 (3): 541-549. doi: 10.1016/j.jse.2019.07.034.

28. Wagner ER, Hevesi M, Houdek MT, Cofield RH, Sperling JW, Sanchez-Sotelo J. Infographic: Replacing failed reverse shoulder arthroplasties as a reliable revision approach. Bone Joint J. 2018; 100-B (11): 1491-1492. doi: 10.1302/0301-620X.100B11.BJJ-2018-1139.

29. Muh SJ, Streit JJ, Lenarz CJ, et al. Resection arthroplasty for failed shoulder arthroplasty. J Shoulder Elbow Surg. 2013; 22 (2): 247-252. doi: 10.1016/j.jse.2012.05.025. 